\title{
Effect of virtual reality aggression prevention training for forensic psychiatric patients (VRAPT): study protocol of a multi-center RCT
}

\author{
Stéphanie Klein Tuente ${ }^{1,2^{*}}$ (D) Stefan Bogaerts ${ }^{3,4}$, Sarah van IJzendoorn ${ }^{4}$ and Wim Veling ${ }^{1}$
}

\begin{abstract}
Background: Many patients residing in forensic psychiatric centers have difficulties regulating their aggression in an adequate manner. Therefore, they are frequently involved in conflicts. Evidenced-based aggression therapies in forensic psychiatry are scarce, and due to the highly secured environment, it is hard to practice real-life provocations. We have developed a Virtual Reality aggression prevention training (VRAPT), providing safe virtual environments, in which patients can practice controlling their aggressive behaviors in an adequate way. The main objective of this study is to examine whether VRAPT is effective in reducing aggression among forensic psychiatric inpatients.
\end{abstract}

Methods: Four forensic psychiatric centers in the Netherlands are participating in this study. Participants will be randomly assigned to either VRAPT or a waiting list. The two groups will be compared at several different time points: baseline (12 weeks before intervention), pre-intervention, post-intervention and at 12 weeks follow-up. After follow-up measurements are completed, participants from the waiting list will also receive VRAPT. The primary outcome is level of aggressive behavior, consisting of staff-reported and self-reported measures. Secondary outcomes are self-report questionnaires on e.g., anger, impulsivity and aggression.

Discussion: To the best of our knowledge this is the first study to examine the effectiveness of a VR aggression prevention training in forensic psychiatric centers. Further details on the methodological issues are discussed in this paper.

Trial registration: Dutch Trial Register (NTR, TC =6340). Retrospectively registered 14-04-2017.

Keywords: Aggression, Aggressive behavior, Virtual reality, Forensic psychiatry, SIP model

\section{Background}

One of the most important issues in working with forensic patients residing in a closed institution is preventing and managing aggressive behavior directed towards staff and other patients [1]. This aggressive behavior not only causes harm to staff and fellow patients, but also has consequences for the treatment progress and the living environment, resulting in longer periods of mandatory treatment. A meta-analysis by Papadopoulos et al.

\footnotetext{
*Correspondence: s.klein.tuente@umcg.nl

'Department of Psychoses, University Medical Center Groningen, Hanzeplein 1, 9713, GZ, Groningen, The Netherlands

${ }^{2}$ Forensic Psychiatric Center (FPC) Dr. S. van Mesdag, Helperlinie 2, 9722, AZ, Groningen, The Netherlands
}

Full list of author information is available at the end of the article showed that almost $40 \%$ of all incidents were preceded by staff-patient interactions. Examples of staff-patient interactions were most frequently denying a patient's request, limiting a patient's freedom or some other restriction [2]. The occurrence of incidents in forensic psychiatric centers (FPCs) is not surprising, as forensic patients often have antisocial (or other) personality disorders, lack of impulse control, a high degree of impulsivity and/or a lack of empathy, all factors that are related to aggression [3, 4]. Accordingly, a majority of patients staying in FPCs are admitted under the judicial measure TBS-order (in Dutch: ter-beschikking-stelling: this translates as 'detained under hospital order'). 'Detained under hospital order' means that the court has 
established a relation between the offense committed and a psychiatric disorder (e.g., [5]). This order applies to serious aggressive offenders having severe psychopathology and who are considered to be at high risk for re-offending.

\section{Aggression}

Aggression is frequently described as having two dimensions [6]. Both dimensions are anchored in the General Aggression Model (GAM; [7]). The GAM is an overarching framework that describes how disruption in cognitive and social processes can lead to aggression. The first dimension, form, concerns the way aggression is expressed: overt (e.g., physical force) or covert (e.g., deliberate manipulation within a relationship). The second dimension, function, relates to the intent of the aggressive behavior [6]. Reactive aggression is an impulsive and under-controlled outburst of anger as a reaction to a perceived or actual threat, provocation or frustration. Proactive aggression refers to a planned or premeditated, controlled display of aggression to achieve personal goals for instance money or power [8,9]. Statistical methods such as factor analysis can distinguish reactive and proactive aggression. Despite this classification, the concept and measurement of aggression remains the object of debate $[9,10]$. Although individuals can engage in both types of aggression, proactive aggression is more often associated with an antisocial and psychopathic personality and with less physiological arousal. Reactive aggression is more commonly related to problems in social information processing; impulsive, anxious or aggressive personality types; and high physiological arousal $[11,12]$.

With regard to the severity and the unpredictable nature of reactive aggression, it is argued that reactive aggression increases the risk of victimization of staff and fellow patients [13]. Therefore, it is important that effective and early interventions focusing on reactive aggression should be top priority for forensic facilities. A systematic review by Ross et al. showed a significant reduction of physically aggressive behavior following an intervention, thereby demonstrating tentative support for the hypothesis that interventions have a positive effect on the reduction of aggressive behavior [14]. Ross et al. discussed research on psychotherapeutic interventions (e.g., Cognitive Behavioral Therapy) for aggressive and violent behavior in forensic populations. Nevertheless, quality assessments of studies included in this review revealed that there were several methodological flaws; overall, most studies were of average quality and none of them included a power analysis [14].

\section{Virtual reality in forensic psychiatry}

Recently, a few studies have examined the use of Virtual Reality (VR) with forensic populations (e.g., [15]), however none of these studies concerned interventions for aggression regulation. Given the positive results from intervention research in other psychiatric disorders, such as social anxiety and PTSD (for a review see [16]), we suggest that VR could also be used for gradual exposure of forensic patients to controlled, provocative virtual social situations. VR exposure can be used to elicit psychological, physiological and behavioral defensive or aggressive responses, to practice new behavior and to test if patients are increasingly able to control their own and others' aggression.

A Virtual Reality Aggression Prevention Training (VRAPT) was designed to create a computer based simulation that shows a virtual environment in which patients are confronted with behaviors and experiences of virtual characters in social situations. VRAPT is based on the Social Information Processing (SIP) model [17]. The rationale of the SIP model is the concept that severe reactive aggression problems are due to problems in social information processing. In VRAPT, the VR components follow the different steps of the SIP model (i.e., encoding, interpretation, goal clarification, response generation, response selection and enactment). These steps as applied in the VRAPT are explained in more detail under heading: "Virtual Reality aggression prevention training (VRAPT)". Furthermore, the VR environments include visual and auditory data and are viewed through stereoscopic glasses. The mechanism of VRAPT is that the patient can engage and practice in de-escalating behavior in interaction with the virtual characters. Practicing allows the patient to learn to recognize imminent aggression of others or himself and to gain control over aggressive impulses. We hypothesize that VRAPT will contribute significantly to the reduction of aggression. Thus leading to reduced aggression both inside and outside FPCs.

\section{Research aims}

The main objective of this study is to investigate the effectiveness of VRAPT in the reduction of reactive aggression in forensic psychiatric inpatients in four Dutch forensic psychiatric centers (FPCs). The primary outcome measure is the level of aggressive behavior, measured by staff-report and a self-report questionnaire. It is expected that both staff-observed and self-reported aggressive behavior will decrease after completion of VRAPT compared to being on the waiting list, and that differences will be maintained after a 12-week follow-up period. Secondary outcome measures are participant's self-reported rates of anger, impulsivity, hostility, physiological arousal, and coping. As all these different components are contributing to the way a patient reacts to provocation of others. 


\section{Design/methods \\ Design}

The study is a single blind randomized controlled trial (RCT) with two conditions: (I) the VRAPT condition, in which participants receive the VR training in addition to treatment as usual (TAU) and (II) the waiting list. Participants on the waiting list receive TAU only and are placed on a waiting list to receive VRAPT after the follow-up measurements have been completed. Participants in both conditions are not allowed to participate in any other therapies or trainings specifically focusing on aggression regulation during the research period. The two conditions are compared at four different periods in time. First, a period of 12 weeks staff observations before intervention (baseline), pre-intervention (T1), post-intervention (T2), and after 12 weeks of staff observations a follow-up (T3). The waiting list is offered VRAPT after the end of T3.

\section{Participants}

All patients exhibiting reactive aggression while residing in the participating FPCs, and are considered eligible after checking the inclusion and exclusion criteria, will be asked to participate (see sample size calculation). The four Dutch FPCs taking part are: FPC Dr. S. van Mesdag, located in Groningen; FPC de Kijvelanden, located in Poortugaal; FPC Pompestichting, located in Nijmegen; FPC de Rooyse Wissel, located in Oostrum. After being informed with an information letter, written informed consent is obtained from each participant.

\section{Inclusion criteria}

Participants are forensic psychiatric patients between age 18-65 referred by their treatment supervisor and/or clinical team for aggression training on the basis of pre-admission history of aggression and current problems with reactive aggression. All DSM-5 diagnoses are included and there are no restrictions with regard to (history of) substance use disorders.

\section{Exclusion criteria}

Exclusion criteria are inability to speak and read Dutch, epilepsy, or mental retardation meaning an IQ below 70 . In case the documented IQ is below 70, but the treatment supervisors indicate that the IQ measurement is not representative for the current state of intellectual functioning, these patients can still be included as participants.

\section{Power and sample size calculation}

There is no previous research using VR aggression regulation training in patient forensic populations based on which an effect size could be estimated. Conventional aggression regulation therapy in such settings has an effect size of around 0.3 [18]. An effect size of 0.5 is considered as moderate. Using this effect size with a $\beta$-power of .80, alpha of .05 and an independent two-sided t-test to evaluate the main outcome, 64 subjects are required in each condition, total $N=128$.

\section{Materials}

An overview of the materials of the study is displayed in Table 1.

\section{Virtual reality aggression prevention training (VRAPT)}

VRAPT consists of 16-biweekly individual training sessions. The VR training protocol is primarily based on the theory of Social Information Processing (SIP; [17]). The SIP model is associated with the General Aggression Model (GAM; [7]. The GAM predicts that aggressive reactions are often both reactive and proactive. As described earlier, VRAPT only covers reactive aggression and therefore we focus on the SIP model.

It is stated that severe reactive aggression problems are caused and remained because of disturbed information processing [17]. Social information processing is a fast and automatic process, involving six different steps that are showed in Fig. 1. This is a simplified version of the original SIP model by Crick and Dodge, so it can also be used as a theoretical model for participants. We transformed the six steps of the model into six questions that can be asked to the participants in different sessions. First encoding: what is going on? Second interpretation: what does this mean? Third selecting a goal: what is the goal I'm trying to achieve in this situation? Fourth generating responses: how can I react to this? Fifth evaluating responses: what am I going to do? And sixth enacting responses: reaction/behavior. These six steps do not only follow each other, but can also influence each other. The center of the circle, including emotions, previous experiences, and physiological stress, all influence the different steps of the SIP model separately.

In the interactive three-dimensional virtual environment, participants have the opportunity to practice new behavior with virtual characters and learning to cope with their own aggressive behavior in an adequate manner. An example of the virtual environment is displayed in Fig. 2. Each step of the SIP model is first practiced separately in VRAPT. Examples include an exercise on recognition of others' facial emotions (i.e., what is going on?); rating the level of aggression of virtual character's behaviors (i.e., what does this mean?); reacting in a sub-assertive manner while being provoked (i.e., how can I react to this?). In the last part of the VRAPT the different exercises will be integrated in more challenging interactive virtual role-plays. Different interactive provocative social scenarios were designed during an iterative process with software engineers, VR experts, clinicians, and researchers. The primary focus of these provocative social scenarios is teaching participants to 
Table 1 Overview of assessments

\begin{tabular}{|c|c|c|c|c|c|c|}
\hline Instrument & 12-week observation (baseline) & Pre-intervention & $\begin{array}{l}\text { During intervention / } \\
\text { waiting list }\end{array}$ & Post-intervention & 12-week observation & Follow-up \\
\hline SDAS & $x$ & & $x$ & & $x$ & \\
\hline AVL & & $x$ & & $x$ & & $x$ \\
\hline CTQ-SF & & $x$ & & & & \\
\hline NAS-PI & & $x$ & & $x$ & & $x$ \\
\hline STAXI-2 & & $x$ & & $x$ & & $x$ \\
\hline BIS-11 & & $x$ & & $x$ & & $x$ \\
\hline $\mathrm{BDHI}-\mathrm{D}$ & & $x$ & & $x$ & & $x$ \\
\hline RPQ & & $x$ & & $x$ & & $x$ \\
\hline HIBT & & $x$ & & $x$ & & $x$ \\
\hline IPQ & & & & $x$ & & \\
\hline Interview (VRAPT only) & & & & & & $x$ \\
\hline
\end{tabular}

SDAS Social Dysfunction and Aggression Scale AVL Aggression Questionnaire CTQ-SF Childhood Trauma Questionnaire - Short Form NAS-PI Novaco Anger Scale and Provocation Inventory STAXI-2 State-Trait Anger Expression Inventory-2 BIS-11 Barratt Impulsiveness Scale BDHI-D Buss-Durkee Hostility Inventory-Dutch RPQ Reactive Proactive Questionnaire HIBT Hostile Intepretation Bias Task IPQ I-Group Presence Questionnaire

cope with their reactive aggression in an adequate manner.

During the VRAPT sessions, participants wear headphones and a head-mounted display while interacting with a virtual character that is controlled by the trainer. The trainer takes the role of the virtual character using a microphone with voice distortion for speech, and also manually controlling facial emotion expression and body movements of the virtual character. This highly dynamic interactive nature of the VR system means VRAPT can be tailored to the specific needs of the participants, and participants have the opportunity to practice with their own learning goals and difficulties. Additionally, real-time galvanic skin response (GSR) and heart rate (HR) will be measured as feedback for participants on their physical arousal during the sessions. Furthermore, trainers will stimulate participants to continue applying

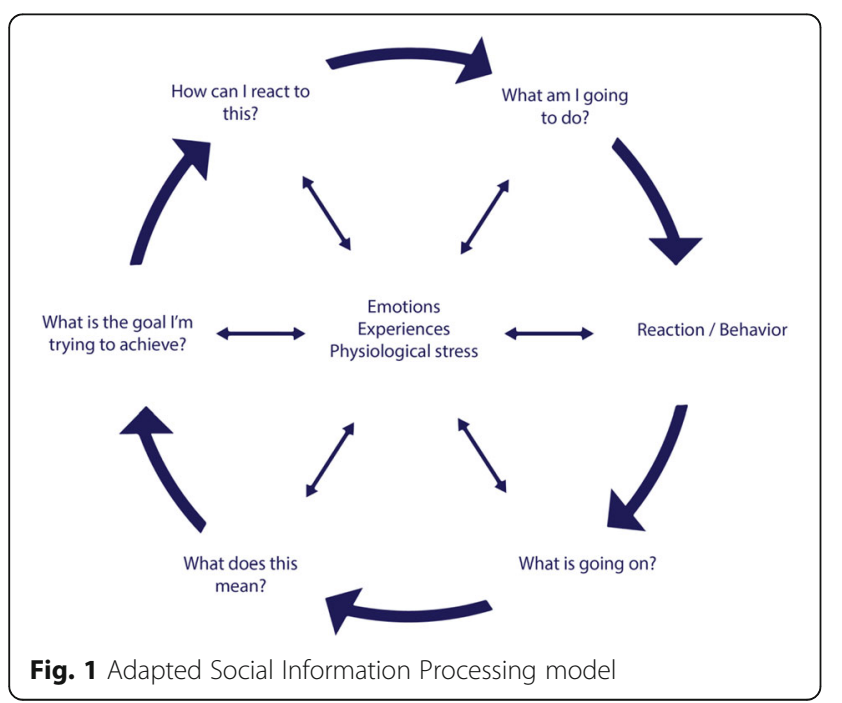

novel behavior they have learnt in VR. At all times the trainer is in control of the virtual environment and is able to immediately change and/or stop the virtual environment if necessary.

\section{Waiting list (control condition)}

Participants in both the VRAPT and waiting list condition receive standard treatment including: supportive counseling by treatment staff, medication and psychological treatment only if it is not directly focused on aggression regulation (allowed e.g., schema therapy, Liberman training, and treatment for addiction problems). Because randomization is on individual level, participants in both conditions may be residing on the same ward and therefore both conditions have access to the same treatment facilities.

\section{Fidelity checks}

The VRAPT protocol is designed as guidance for the trainers and to ensure treatment integrity. Each session in the protocol follows the same format: short discussion of previous session, clarification of the step of the SIP model, conversation about the VR assessment, technical guidance and each session ends with an evaluation of the learning goals. Besides, VRAPT-trainers receive $16 \mathrm{~h}$ of training in using the VRAPT system and the protocol. The training is provided by the first and third author, respectively the researcher and a highly skilled psychologist. In addition to this training, monthly one-hour group multicenter videoconference supervision serves to guide the trainers throughout the intervention period and is facilitated by one of the two clinical experts; the third and last author. Furthermore, during the intervention, trainers are required to complete session forms, which are checked for completeness by the research 


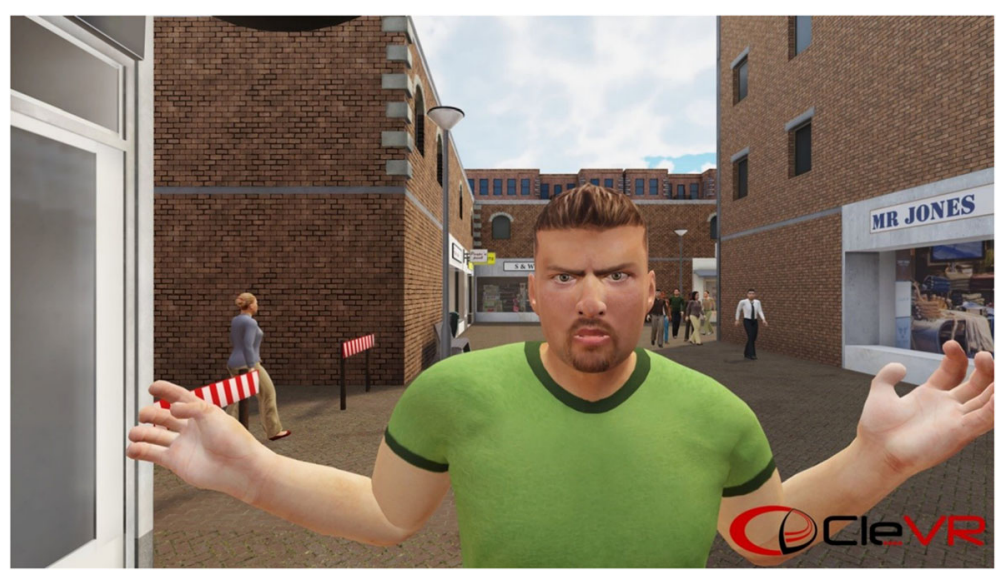

Fig. 2 Impression of the virtual environment

assistant afterwards. Finally, at two different moments during the VRAPT sessions, trainers have to send their learning goals to a highly skilled clinician within their own organization for feedback.

\section{Measurement instruments Primary outcome}

Level of aggressive behaviour Primary outcome measure is level of aggressive behaviour. Self-report questionnaires and staff-reports are used to assess the effectiveness of VRAPT, because the perspectives of patients and staff represent distinct but complementary measures of aggressive behaviour.

The social dysfunction and aggression scale (SDAS) Staff is trained and asked to complete the Social Dysfunction and Aggression Scale (SDAS; [19, 20]) weekly for each patient meeting the inclusion criteria and signed informed consent, to document the aggressive state of patients. SDAS data is collected at least 12 weeks before VRAPT starts. The SDAS provides systematic recording of staff observations on a broad range of aggressive behaviour on a weekly basis. In this research, the 9-item version was chosen. Illustrative examples are: irritability, e.g., difficulty controlling reactions; negativism, e.g., not wanting to cooperate; directed verbal aggressiveness, e.g., insulting people personally. All 9 items are scored along a 4-point scale ranging from absent to severely present.

In the current study, the Dutch SDAS manual of Bousardt is used. As described in this manual, for each of the items a general and peak score can be scored [21]. In this way, each SDAS item is scored twice, first on the most severe aggressive behaviour (peak), and second on average level of aggressive behaviour. SDAS scores of participants in the VRAPT condition (intervention group) will be compared with the SDAS scores of participants on the waiting list (control group). Psychometric properties of the Dutch version of the SDAS are moderate inter-rater reliability and good convergent validity in a FPC (Cronbach's $\alpha$ (11 items) = .82; [20]).

Aggression questionnaire (AVL) In addition, participants are asked to the Dutch version of the Aggression Questionnaire (AVL; [22]) at three different measuring points: pre-intervention (T1), post-intervention (T2), and 12-week follow-up (T3). This questionnaire assesses four sub traits of aggression, i.e., physical aggression, verbal aggression, anger and hostility [23]. The test-retest reliability of the AQ total scores were good and validity showed significant correlations with alternative aggression measurements. A Dutch study showed good internal consistency (Cronbach's $\alpha$ (total, inpatients) $=.83 ;[24])$.

\section{Secondary outcome measures}

Components of the SIP model are used as secondary outcomes and are assessed with diverse self-report questionnaires.

Child trauma questionnaire-short form (CTQ-SF) The Child Trauma Questionnaire-Short Form (CTQ-SF; [25]) is used to measure childhood neglect and abuse, which are predictive factors for victimization and being a perpetrator in adulthood [26]. Moreover, male prisoners scoring high on the CTQ-SF were significantly more often involved in violent behaviour during their stay in prison [27]. The Dutch version of the CTQ-SF demonstrated adequate internal consistency reliability in a large community sample including amongst other patient groups forensic patients and prisoners (Cronbach's $\alpha \geq .63$; [28]). 
Barratt impulsiveness scale (BIS-11) The Barratt Impulsiveness Scale (BIS-11; [29] is a 30-item measurement used to study the role of impulse control [30]. The BIS-11 has three subscales, namely: attentional impulsiveness, non-planning impulsiveness, and motor impulsiveness. A study showed that the BIS-11 is sensitive to different levels of aggression [31]; non-violent offenders scored lower on the BIS-11 than their violent counterparts (Cronbach's $\alpha=.81$; [32]).

Buss-Durkee hostility inventory-Dutch (BDHI-D) The Buss-Durkee Hostility Inventory-Dutch (BDHI-D; [33]) is used to measure two factors of aggression: covert or indirect aggression (20 items) and overt or direct aggression (20 items), and is rated on a 'true'- 'not true' dichotomous scale [34]. The combination of verbal and physical aggression represents direct aggression, whereas hostility and anger are the core concepts of indirect aggression (Cronbach's $\alpha$ (Overt Aggression) $=.77, \alpha(\mathrm{Cov}-$ ert Aggression) = .79; [34]).

\section{Novaco anger scale and provocation inventory (NAS-} PI) The Novaco Anger Scale and Provocation Inventory (NAS-PI; [35]) is a two-part test of 73 items designed to assess anger as a problem of psychological functioning and physical health and to assess therapeutic change [35]. In Dutch forensic psychiatric patient samples, the internal consistency of the NAS-PI is excellent (Cronbach's $\alpha$ (NAS, total $) \geq .92$, $\alpha$ (PI, inpatients $)=.90$ ). Furthermore, in a forensic outpatient sample the test-retest reliability was good [35].

State-trait anger expression Inventory-2 (STAXI-2) The State-Trait Anger Expression Inventory-2 (STAXI-2; [36]) is used to measure the experience, expression, and control of anger. The STAXI-2 is a 57 -item measure with scales developed to assess anger on three different scales: 1) state anger scale, this is situational anger; 2) trait anger scale, this is a dispositional characteristic, and 3 ) anger expression scale, this is the expression of anger [37]. The Dutch version of the STAXI-2 has adequate psychometric properties (Cronbach's $\alpha \geq .71$; [38]).

Reactive-proactive questionnaire (RPQ) Reactive-Proactive Questionnaire (RPQ) is a self-report of aggression that is not restricted to a short period of time. The RPQ is a questionnaire in which the person is asked about the reasons and the type of aggressive behaviour and refers to this behaviour in general [39]. The RPQ has an excellent internal consistency (Cronbach's $\alpha .91 ;$ [40]).

Hostile interpretation Bias task (HIBT) Hostile Interpretation Bias Task (HIBT) is a 10-min computer task to measure hostile interpretation of ambiguous facial expressions of emotions [41].

I-group presence questionnaire The Igroup Presence Questionnaire (IPQ) is used to measure sense of presence in the virtual environment. The IPQ consists of 14 items, responses are made on a 7-point Likert scale, and it has demonstrated good psychometric properties [42].

Interview At follow-up, patients in the VRAPT condition will be interviewed by the research assistant to assess their own opinion about VRAPT. Questions such as: "how did VRAPT contribute to your personal aggression management?" will be asked. This will contribute to the secondary outcome measures, as specific questions about VRAPT can be asked in addition to the self-report questionnaires.

\section{Procedure}

All patients are screened by their treatment supervisors based on the in- and exclusion criteria. Patients who meet inclusion criteria are invited by an independent research assistant to participate, and receive an information letter (Fig. 3). Patients have 1 week to consider participation, after which written informed consent is obtained. Next, participants are randomly allocated to either VRAPT or waiting list condition and are monitored with the SDAS by staff for aggression on a weekly basis during 12 weeks before start of the intervention until the end of the follow-up. Before the intervention starts, pre-intervention measures are obtained (see Table 1). During 10 out of 16 sessions of VRAPT physiological measures of emotional arousal (i.e., heart rate and galvanic skin response) are measured. VRAPT consists of 16 bi-weekly individual sessions with a maximum duration of 60 min per session. The participants in the control condition will be placed on a waiting list. They will have the same assessments as the VRAPT group, i.e., the 32-weeks SDAS, pre-intervention, post-intervention and follow-up measurements. They will receive VRAPT after the last follow-up measurement. During the study period, for both conditions, treatment as usual will proceed, except for specific anger or aggression management therapy. During the intervention, aggression keeps being monitored with the SDAS by staff working on the ward. After the last session of the intervention, pre-intervention self-report measures are repeated in all participants, guided by assessors who are blinded for treatment allocation. During 12 weeks after end of the intervention, participants keep being monitored for aggressive behavior. Twelve weeks after the last session, self-report measures are repeated again. 


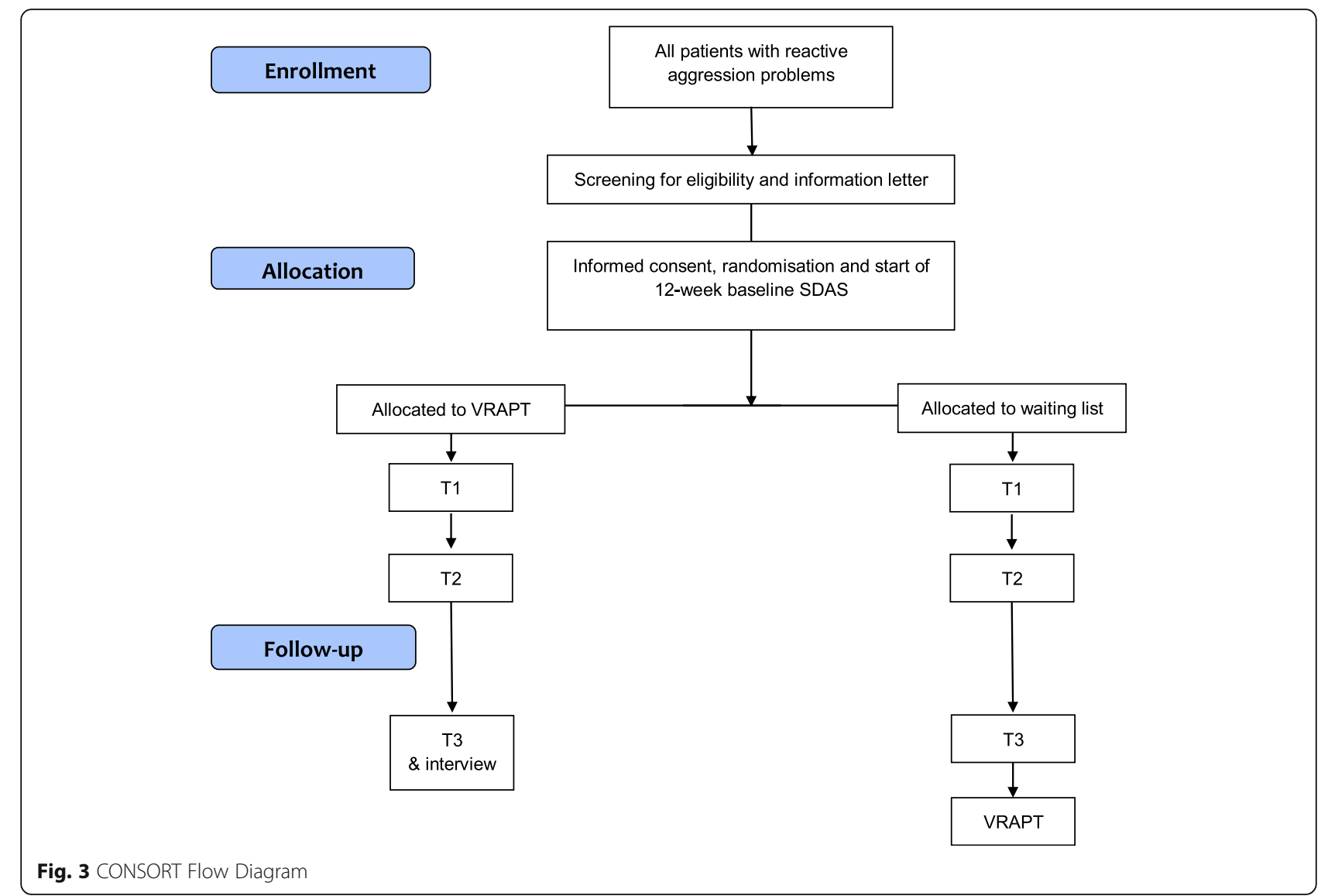

\section{Randomization}

Participants will be randomly assigned to one of two conditions: VRAPT or waiting list. Randomization is conducted via a scientific randomization program on the Internet (https://www.randomizer.org), by the research coordinator of the Department of Psychiatry from the University Medical Center Groningen. Twenty-two sets consisting of two unique numbers are made available for each participating FPC. Research assistants are informed about the randomization by e-mail and communicate the condition verbally to the participants and the clinical teams.

\section{Statistical analysis}

Analysis of the data will be conducted according to the intention to treat principle [43]. The result of randomization will be checked by comparing baseline socio-demographic and clinical parameters between VRAPT and the waiting list condition, using univariate analyses (chi-square tests for dichotomous measures and $\mathrm{t}$-tests for continuous measures). Differences in scores between pre-intervention, post-intervention and follow-up assessments and between intervention and control group will be examined for the dependent variables, using multilevel (repeated-measures) random linear regression analysis.
Primary outcome measure is peak and general score as measured with the SDAS and self-report aggression measurement of participants. The SDAS and self-report measurement will be compared between VRAPT and waiting list, before, during and after VRAPT or waiting list. Effects of VRAPT will be tested with group $\mathrm{x}$ time interaction terms. Risk factors for aggression will be used as covariates in the linear regression models. Significance of fixed effects are assessed by Likelihood Ratio tests. Statistical significance of the regression effects will be tested using T-tests. In all analyses, a $p$-value $<.05$ will be considered statistically significant.

\section{Discussion}

The main goal of this study is to investigate the effectiveness of VRAPT on reactive aggressive behavior of forensic psychiatric inpatients. This study will be the first to evaluate the effectiveness of a VR intervention targeted at reducing reactive aggressive behavior of forensic patients. The hypothesis of the study is that VRAPT will decrease both self-reported and staff-reported aggressive behavior.

Inpatient aggressive behavior is a predictor for future aggression in society, because inpatient aggression is related to non-compliance, higher treatment dropout, and 
more personality disorders [44]. Current interventions focusing on the reduction of aggressive behavior have several limitations [18]. First, exposure to provocation in highly secured forensic settings is limited. Thus, it is not possible to practice and train forensic patients to control the anger of others and themselves by provoking them in real life social situations. Second, social skill training to prevent aggression by others is limited, and is usually practiced only in treatment groups and role play. Third, engaging forensic patients in treatment is challenging. Attrition is high, as many of them do not like treatment, and/or find it difficult to apply cognitive therapeutic insights. Fourth, it is difficult to evaluate objectively and reliably to what extent patients have learnt to regulate their aggressive impulses, which makes forensic risk assessment complicated.

Addressing the previously listed limitations, VRAPT has several advantages. First, patients can have the opportunity to practice with difficult behavior and additionally gain tools to cope with aggression, instead of leaving the situation or avoiding angry emotions. Second, VR is an interactive tool that allows trainers to provoke and trigger aggression during the training. Often this is described as a difficult aspect by trainers, however, with VRAPT the trainers feel safer in provoking participants, as the participant faces the virtual character and the trainer's voice is transformed. This also works the other way around: because the trainer 'plays' as the virtual character, it is safer for the participant to express his aggression without worrying about the therapeutic relationship. Third, VRAPT is an experiential, behavioral training, focusing on practicing behavior and not necessarily on gaining cognitive therapeutic insights. Fourth, objective and reliable assessment of aggression in real life situations on treatment wards is difficult. Therefore, in the VRAPT real-time measurements of physiological arousal are included. These measurements help to gain more insight into arousal of the patients. Moreover, not only self-report measurements, but also staff observations are recorded. All these aspects are of specific importance because forensic psychiatric patients are at an increased risk of behaving in an aggressive manner.

From a broader perspective, this study will shed light on the effectiveness of VR interventions in forensic populations. If proven to be effective, VRAPT could be a useful addition to current interventions in forensic psychiatry and be implemented in other forensic psychiatric centers, especially as evidence based interventions to reduce aggressive behavior are scarce [14]. In addition, reducing aggression of forensic patients is of great importance for patients, but also for staff working in forensic psychiatric centers, as many fellow patients and treatment staff are victimized by aggression of forensic patients. Because patients can practice safely with difficult situations that they can expect to be confronted with in the outside world, VRAPT may be an important contribution to the preparation for reintegration into society. Thus VRAPT is expected to lead to improvement of psychological, social and emotional well-being of patients and treatment staff.

\section{Trial status \\ Ongoing.}

\begin{abstract}
Abbreviations
AVL: Aggression Questionnaire; BDHI-D: Buss-Durkee Hostility InventoryDutch; BIS-11: Barratt Impulsiveness Scale; CTQ-SF: Childhood Trauma Questionnaire - Short Form; DSM: Diagnostic and Statistical Manual of Mental Disorders; FPCs: Forensic Psychiatric Centers; GAM: General Aggression Model; GSR: galvanic skin response; HIBT: Hostile Interpretation Bias Task; HR: heart rate; IPQ: Igroup Presence Questionnaire; IQ: Intelligence quotient; NAS-PI: Novaco Anger Scale - Provocation Inventory; NTR: Dutch Trial Register; NWO: Netherlands Organization for Scientific Research; PTSD: Post-Traumatic Stress Disorder; RCT: Randomized Controlled Trial; RPQ: Reactive-Proactive Questionnaire; SDAS: Social Dysfunction and Aggression Scale; SIP model: Social Information Processing model; STAXI2: State-Trait Anger Expression Inventory-2; TAU: Treatment As Usual; TBSorder in Dutch: ter-beschikking-stelling: this translates as 'detained under hospital order'; VR: Virtual Reality; VRAPT: Virtual Reality Aggression Prevention Training
\end{abstract}

\section{Acknowledgments}

We would like to thank the participating forensic psychiatric centers: FPC de Kijvelanden, FPC Dr. S. van Mesdag, FPC Pompestichting, FPC de Rooyse Wissel and the trainers and experts participating in this trial. We also like to acknowledge research coordinator Erna van 't Hag.

\section{Funding}

The study is funded by the Netherlands Organization for Scientific Research, as part of the research programme "Geweld tegen psychatrisch patienten" (Violence against psychiatric patients; NWO grant nr 432-13-802).

\section{Authors' contributions}

WW and SB conceived and designed the study. WV, SB, SKT, SVIJ, developed the VRAPT training protocol. SKT wrote the first draft of the manuscript. SB, SvIJ, WV critically revised the manuscript. All authors have read and approved the final manuscript.

\section{Ethics approval and consent to participate}

The protocol of this study has been approved by the medical ethical board of the University Medical Centre Groningen, Groningen (number: NL52939.042.15). The study will be conducted in accordance with the principles of the Declaration of Helsinki. Written informed consent to participate in the study will be obtained from all the participants.

Consent for publication

Not applicable.

\section{Competing interests}

The authors declare that they have no competing interests.

\section{Publisher's Note}

Springer Nature remains neutral with regard to jurisdictional claims in published maps and institutional affiliations.

\section{Author details}

'Department of Psychoses, University Medical Center Groningen, Hanzeplein 1, 9713, GZ, Groningen, The Netherlands. ${ }^{2}$ Forensic Psychiatric Center (FPC)

Dr. S. van Mesdag, Helperlinie 2, 9722, AZ, Groningen, The Netherlands.

${ }^{3}$ Department of Developmental Psychology, Tilburg University, Prof

Cobbenhagenlaan 225, PO Box 90153, 5000, LE, Tilburg, The Netherlands. 
${ }^{4}$ Fivoor, Fivoor Science \& Treatment Innovation, Kijvelandsekade 1, 3172, AB, Poortugaal, The Netherlands.

\section{Received: 3 February 2018 Accepted: 25 July 2018 Published online: 06 August 2018}

\section{References}

1. Novaco RW, Taylor JL. Behaviour research and therapy reduction of assaultive behavior following anger treatment of forensic hospital patients with intellectual disabilities. Behav Res Ther. 2015;65:52-9.

2. Papadopoulos C, Ross J, Stewart D, Dack C, James K, Bowers L. The antecedents of violence and aggression within psychiatric in-patient settings. Acta Psychiatr Scand. 2012:125(6):425-39.

3. Bogaerts S, Polak M, Spreen M, Zwets A. High and low aggressive narcissism and anti-social lifestyle in relationship to impulsivity, hostility, and empathy in a Group of Forensic Patients in the Netherlands. J Forensic Psychol Pract. 2012;12(2):147-62

4. Lobbestael J, Cima M, Lemmens A. The relationship between personality disorder traits and reactive versus proactive motivation for aggression. Psychiatry Res. 2015;229(1-2):155-60.

5. van Marle HJC. The Dutch entrutstment act (TBS) its principles and innovations.Pdf. Int J Forensic Ment Health. 2002;1(1):83-92.

6. Bailey CA, Ostrov JM. Differentiating forms and functions of aggression in emerging adults: associations with hostile attribution biases and normative veliefs. J Youth Adolesc. 2008;37(6):713-22.

7. Anderson CA, Bushman BJ. Human Agression. Annu Rev Psychol. 2002;53: 27-51.

8. Dodge KA, Coie JD. Social-information-processing factors in reactive and proactive aggression in children's peer groups. J Pers Soc Psychol. 1987; 53(6):1146-58

9. Poulin F, Boivin M. Reactive and proactive aggression: evidence of a twofactor model. Psychol Assess. 2000;12(2):115-22.

10. Raine $\mathrm{A}$, et al. The reactive-proactive aggression questionnaire: differential correlates of reactive and proactive aggression in adolescent boys. Aggress Behav. 2006:32(2):159-71.

11. Cima M, Raine A. Distinct characteristics of psychopathy relate to different subtypes of aggression. Pers Individ Dif. 2009:47(8):835-40.

12. Scarpa A, Tanaka A, Chiara Haden S. Biosocial bases of reactive and proactive aggression: the roles of community violence exposure and heart rate. J Community Psychol. 2008;36(8):969-88.

13. Steadham JA, Rogers R. Predictors of reactive and instrumental aggression in jail detainees: an initial examination. J Forensic Psychol Pract. 2013;13(5):411-28.

14. Ross J, Quayle E, Newman E, Tansey L. The impact of psychological therapies on violent behaviour in clinical and forensic settings: a systematic review. Aggress Violent Behav. 2013;18(6):761-73.

15. Benbouriche M, Nolet K, Trottier D, Renaud P. Virtual reality applications in forensic psychiatry. In Proceedings of the Virtual Reality International Conference. Laval: Laval Virtual (VRIC '14); 2014.

16. Valmaggia LR, Latif $L$, Kempton MJ, Rus-Calafell M. Virtual reality in the psychological treatment for mental health problems: an systematic review of recent evidence. Psychiatry Res. 2016;236:189-95.

17. Crick NR, Dodge KA. A review and reformulation of social informationprocessing mechanisms in Children's social adjustment. Psychol Bull. 1994; 115(1):74-101.

18. McGuire J. A review of effective interventions for reducing aggression and violence. Philos Trans R Soc Lond Ser B Biol Sci. 2008;363(1503):2577-97.

19. Wistedt $B$, et al. The development of an observer scale for measuring social dysfunction and aggression. Pharmacopsychiatry. 1990;23:249-52.

20. Kobes MHBM, Nijman HHLI, Bulten EBH. Assessing aggressive behavior in forensic psychiatric patients: validity and clinical utility of combining two instruments. Arch Psychiatr Nurs. 2012;26(6):487-94.

21. Bousardt A, Hoogendoorn AW, Noorthoorn EO, Hummelen JW, Nijman HL. Predicting inpatient aggression by self-reported impulsivity in forensic psychiatric patients. Crim Behav Ment Health. 2016;26(3):161-73.

22. Meesters C, Muris P, Bosma H, Schouten E, Beuving S. Psychometric evaluation of the Dutch version of the Aggression Questionnaire. Behav Res Ther. 1996;34(10):839-43

23. Buss AH, Perry M. The Aggression Questionnaire. J Pers. 1992;63(3):452-9.

24. Hornsveld RHJ, Muris P, Kraaimaat FW, Meesters C. Psychometric properties of the aggression questionnaire in Dutch violent forensic psychiatric patients and secondary vocational students. Assessment. 2009;16(2):181-92.
25. Bernstein DP, et al. Development and validation of a brief screening version of the childhood trauma questionnaire. Child Abus Negl. 2003:27(2):169-90.

26. Kamperman AM, et al. Criminal victimisation in people with severe mental illness: a multi-site prevalence and incidence survey in the Netherlands. PLoS One. 2014;9(3):1-13.

27. Sarchiapone M, Carli V, Cuomo C, Marchetti M, Roy A. Association between childhood trauma and aggression in male prisoners. Psychiatry Res. 2009; 165(1-2):187-92.

28. Thombs BD, Bernstein DP, Lobbestael J, Arntz A. A validation study of the Dutch childhood trauma questionnaire-short form: factor structure, reliability, and known-groups validity. Child Abus Negl. 2009:33(8):518-23.

29. Patton JH, Stanford MS, Barratt ES. Patton factor structure of the BIS.Pdf. J Clin Psychol. 1995:51(6):768-74.

30. Reise SP, Moore TM, Sabb FW, Brown AK, London ED. The barratt impulsiveness scale-11: reassessment of its structure in a community sample. Psychol Assess. 2013;25(2):631-42.

31. Smith $P$, Waterman $M$, Ward N. Driving aggression in forensic and nonforensic populations: relationships to self-reported levels of aggression, anger and impulsivity. Br J Psychol. 2006;97(3):387-403.

32. Goudriaan AE, Oosterlaan J, De Beurs E, Van Den Brink W. The role of selfreported impulsivity and reward sensitivity versus neurocognitive measures of disinhibition and decision-making in the prediction of relapse in pathological gamblers. Psychol Med. 2008;38(1):41-50.

33. Lange A, Hoogendoorn M, Wiederspahn A, de Beurs E. BDHI-Dutch (BussDurkee Hostility Inventory): Handleiding, verantwoording en normering van de Nederlandse Buss-Durkee agressievragenlijst. Lisse: Swets Test Services; 1995.

34. Lange A, Pahlich A, Sarucco M, Smits G, Dehghani B, Hanewald G. Psychometric characteristics and validity of the Dutch adaptation of the Buss-Durkee hostility inventory (the BDHI-D). Behav Res Ther. 1995;33(2): $223-7$.

35. Hornsveld RHJ, Muris P, Kraaimaat FW. The Novaco anger scale-provocation inventory (1994 version) in Dutch forensic psychiatric patients. Psychol Assess. 2011;23(4):937-44.

36. Hovens JE, Lievaart M, Rodenburg JJ. STAXI-2: Vragenlijst over boosheid. Amsterdam: Hogrefe; 2014

37. Spielberger CD, Sydeman SJ. State-Trait Anxiety Inventory and State-Trait Anger Expression Inventory. In: The use of psychological testing for treatment planning and outcome assessment. Hillsdale, NJ, US: Lawrence Erlbaum Associates, Inc; 1994. p. 292-321.

38. Lievaart M, Franken IHA, Hovens JE. Anger assessment in clinical and nonclinical populations: further validation of the state-trait anger expression Inventory-2. J Clin Psychol. 2016;72(3):263-78.

39. Domburgh L, Popma A. Reactive and proactive questionnaire [Dutch translation]. Duivendrecht, The Netherlands; 2003.

40. Cima M, Raine A, Meesters C, Popma A. Validation of the Dutch reactive proactive questionnaire (RPQ): differential correlates of reactive and proactive aggression from childhood to adulthood. Aggress Behav. 2013; 39(2):99-113.

41. Smeijers D, Rinck M, Bulten E, van den Heuvel T, Verkes RJ. Generalized hostile interpretation bias regarding facial expressions: characteristic of pathological aggressive behavior. Aggress Behav. 2017;43(4):386-97.

42. Schubert $T$, Friedmann F, Regenbrecht $H$. The experience of presence: factor analytic insights. Presence. 2001;10(3):266-81.

43. Altman DG, et al. The revised Consort statement for reporting randomized trials: explanation and elaboration (2001) (ending). Ann Intern Med. 2001; 134(8):663-94.

44. Jeandarme I, Wittouck C, Vander Laenen F, Pouls C, Oei TI, Bogaerts S. Risk factors associated with inpatient violence during medium security treatment. J Interpers Violence. 2016:1-26. http://journals.sagepub.com/doi/ pdf/10.1177/0886260516670884. 